\title{
Disease Tolerance as a Defense Strategy
}

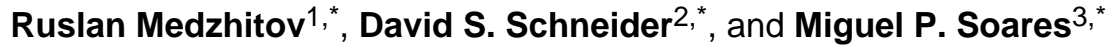 \\ ${ }^{1}$ Howard Hughes Medical Institute, Department of Immunobiology, Yale University School of \\ Medicine, New Haven, CT \\ ${ }^{2}$ Department of Microbiology and Immunology, Stanford University, Palo Alto, CA \\ ${ }^{3}$ Instituto Gulbenkian de Ciência, Oeiras, Portugal
}

\begin{abstract}
The immune system protects from infections primarily by detecting and eliminating the invading pathogens; however, the host organism can also protect itself from infectious diseases by reducing the negative impact of infections on host fitness. This ability to tolerate a pathogen's presence is a distinct host defense strategy, which has been largely overlooked in animal and human studies. Introduction of the notion of "disease tolerance" into the conceptual toolkit of immunology will expand our understanding of infectious diseases and host pathogen interactions. Analysis of disease tolerance mechanisms should provide new approaches for the treatment of infections and other diseases.
\end{abstract}

\section{Introduction}

Animal host defense mechanisms have traditionally been thought to be a function of the immune system that aims to detect and eliminate invading pathogens. However, ecological analyses have long described a genetic variation in disease susceptibility in plants that could be dissociated from their ability to control pathogen burden $(1,2)$. This variation is due to differential ability to tolerate the presence of pathogens, and tolerance to infection was thus realized to constitute a distinct strategy of host defense. The concept of tolerance was only recently introduced into the field of animal immunity (3-6) and concerted efforts will be required to fully elucidate the role of tolerance in host protection from infectious diseases.

In principle, the host can protect itself from infectious disease using three distinct strategies: avoidance, resistance and tolerance. Avoidance reduces the risk of exposure to infectious agents. Resistance reduces pathogen burden once the infection is established. Tolerance reduces the negative impact of an infection on host fitness without directly affecting the pathogen burden $(3,5,6)$. The term tolerance used in this context is not to be confused with immunological tolerance, which is defined as unresponsiveness to self antigens. In some contexts, however, the two terms can be used interchangeably because immunological tolerance is a special case of a multitude of mechanisms that protect the host from immune or pathogen inflicted damage.

\section{Avoidance}

The avoidance strategy works through alteration of host behavior and requires that the host detects the risk of pathogen exposure before being infected. Sensing pathogens prior to infection is mediated primarily through the olfactory and gustatory systems, although visual

\footnotetext{
*To whom correspondence should be addressed: ruslan.medzhitov@yale.edu, dschneider@stanford.edu, mpsoares@igc.gulbenkian.pt.
} 
cues can also be used in some species. Pathogen presence in the environment is detected through various molecular proxies of high pathogen density, such as volatile metabolites specifically produced by microorganisms, including pathogens. For example, cadaverine, putrescine and skatole (3-methylindole), are chemicals produced by bacterial metabolism of amino acids that occurs during putrefaction of animal tissues. Methane thiol is produced by bacterial breakdown of L-methionine and contributes to the characteristic body odor associated with high bacterial densities on the skin. At high doses, these chemicals have foul odor (as perceived by humans) and thus report on high bacterial densities and therefore high risk of infection. Their detection by the olfactory system triggers aversive behavior (at least in some species) that helps to reduce the risk of infection (7). For example, a subset of formyl-peptide receptors is expressed in the mammalian vomeronasal organ where they function as olfactory receptors and presumably detect pathogens or infected conspecifics ( 8 , 9). The gustatory sensory system is also involved in triggering aversive behaviors and reflexes. Interestingly, the chemosensory system used to sense bitter taste also appears to be used to detect acyl-homoserine lactone, a bacterial quorum sensing molecule that signals high bacterial density (10). It is unclear to what extent different aversive behaviors are innate or learned; this likely depends on the stimulus and the host species. The mechanism of aversive behavior is best understood in Caenorhabditis elegans, where avoidance of pathogens is a learned behavior, mediated by the olfactory neurons $(11,12)$. Social insects also have well documented avoidance behaviors that help minimize colony exposure to pathogens (13). Bullfrog tadpoles use chemical cues to detect and avoid infected conspecifics (14). In rodents, detection of infected conspecifics through the olfactory system controls mate selection and avoidance of social contact (7). Aversive behavior and feelings of disgust also play an important role in humans, helping to reduce pathogen exposure (15). Although pathogen avoidance can have a clear adaptive value, extreme forms of aversive behavior can be a significant handicap, as exemplified by germophobia, a common type of obsessive-compulsive disorder.

\section{Resistance}

Resistance mechanisms protect the infected host by reducing its pathogen burden.

Resistance is a function of the immune system, which works by detection, neutralization, destruction or expulsion of the pathogens. Both innate and adaptive immune systems contribute to host resistance to infections. Although the resistance strategy is clearly crucial for host protection from infections, it carries a significant cost to host fitness (16).

Destruction and elimination of pathogens is often accompanied by collateral tissue damage. Even in the absence of overt tissue damage, resistance mechanisms commonly occur at a cost to normal tissue function. For example, infection-induced inflammation alters normal vascular function and increased permeability of airway epithelium can compromise normal respiratory function (17). Collectively, the negative impact of immune defenses on host fitness are referred to as immunopathology (18). Immunopathology is an unavoidable consequence of immune defenses. In general, the degree of immunopathology is positively correlated with the magnitude and duration of the immune response. Consequently, the trade-off between immunity and immunopathology constrains both the evolution and the deployment of resistance mechanisms. Thus the optimal immune response is determined by the balance between efficient pathogen clearance and an acceptable level of immunopathology (19). Because insufficient immunity results in high rate of mortality from infections, the acceptable level of immunopathology can be high, making it a common cause of infectious disease symptoms. The trade-off between protective immunity and immunopathology can be de-constrained, to an extent, by the tolerance mechanisms, which limit tissue damage thus allowing for a higher magnitude and duration of the immune response than would have been otherwise possible. 


\section{Tolerance}

Tolerance is a host defense strategy that reduces the negative impact of infection on host fitness. Unlike resistance mechanisms, tolerance does not directly affect pathogen burden. Rather, tolerance decreases the host susceptibility to tissue damage, or other fitness costs, caused by the pathogens or by the immune response against them (Fig. 1).

The concept of tolerance as a defense strategy has been developed and used in studies of plant immunity for decades $(1,2)$. However, it was only recently introduced into the field of animal immunity $(3,5,6)$. The original observation by Raberg et al (4) demonstrated that disease severity in Plasmodium infected mice can be dissociated from pathogen burden, thus providing the first clear example where this ecological definition of host tolerance was applied to infections in animals. This study also demonstrated differences in tolerance between inbred mouse strains, demonstrating that tolerance is genetically determined. Tolerance as a common host defense strategy in animals was demonstrated by a genetic screen of flies. Using survival to a lethal bacterial infection as a readout, tolerance mutants were isolated as frequently as resistance mutants (20). The subsequent study demonstrated that the balance of tolerance and resistance was pathogen specific and that a set of conditions that might favor tolerance for one pathogen could promote resistance against another (21). A similar phenomenon was described in mice, where tolerance to plasmodium was found to compromise the resistance against Salmonella infection (22). Another study in Drosophila identified a phagocytosis-dependent tolerance mechanism (23), illustrating involvement of the same process in both resistance and tolerance. Studies in C. elegans demonstrated that the unfolded protein response (UPR) plays a role in promoting tolerance to Pseudomonas aeruginosa infection, providing an example of how a stress response can help a host endure an infection (24). Interestingly, this UPR in worms can be triggered systemically through a neuronal mechanism, illustrating that the nervous system can play a role in tolerance to infection (25).

In mice, tolerance mechanisms have been best characterized in experimental models of Plasmodium infection, the causative agent of malaria. The blood stage of Plasmodium infection is associated with hemolysis and the release of hemoglobin. Cell-free hemoglobin promotes tissue damage and organ failure and its toxic effect does not scale with the Plasmodium burden $(26,27)$. This suggests that, within a certain range, protecting the tissues from the toxic effect of hemoglobin can promote host fitness and survival independently of pathogen load. Indeed this was shown to be the case $(28,29)$. Tolerance to Plasmodium infection is conferred by a mechanisms involving heme oxygenase-1 (HO-1), encoded by a stress inducible gene Hmox 1, which is induced in response to oxidative stress $(29,30)$. Interestingly, sickle cell mutations, selected in areas where malaria is endemic, confer tolerance to Plasmodium infection via a genetic program involving inducible $\mathrm{HO}-1$ expression (28). The same pathway is also essential to promote tolerance to severe sepsis, illustrating the broad role of stress responsive genes in host tolerance (31).

Currently, very little is known about the full spectrum of tolerance mechanisms and the few available studies in animals cited above already hint at their diversity in different species and in different types of infection. Because pathogens and immunopathology can potentially affect almost any physiological process, tolerance is not restricted to a single protective pathway, but rather encompasses multiple mechanisms that help reduce the host vulnerability to damage.

\section{Infection-associated fitness costs}

During an infection the host can sustain two types of tissue damage: direct damage by the pathogen and immunopathology. Accordingly, the host can employ two types of tolerance 
mechanisms, one minimizing direct pathogen-induced damage, the other minimizing immunopathology. Both pathogens and the immune response can cause tissue damage by a variety of mechanisms: they can directly kill infected cells or disrupt normal tissue architecture, homeostasis and function. Therefore, protection from infection-induced tissue damage in principle can be achieved by multiple mechanisms. For example, metaplasia of columnar epithelium into stratified squamous epithelium or hyperplasia and increased mucus production by goblet cells will both decrease susceptibility to epithelial damage by pathogens or other environmental insults (17). Other types of increased tissue protection will have a similar effect. Efficient repair of damaged tissues and adaptation to the consequences of tissue damage, such as hypoxia, can also increase the level of tolerance. Thus, efficient replacement of damaged red blood cells through induced erythropoiesis may help tolerate infections with hemolytic pathogens. Clearly, the same mechanism would not be effective in the case of infections that do not cause anemia. In general, different infections cause different spectra of tissue damage and these can, in turn, be ameliorated by different tolerance mechanisms. Increased tolerance to tissue damage can be achieved, in general, through tissue protection and repair.

Although tissue damage caused by pathogens or by the immune response is the most obvious negative outcome of an infectious disease, overt tissue damage is only one of many possible fitness costs associated with infections. In principle, almost any physiological process can be negatively affected by pathogens or by the immune and inflammatory responses they elicit. Although specific tolerance mechanisms employed in these cases are largely unknown, they would be expected to prevent, reduce or counter the pathological alterations caused by infections. Therefore, the mechanisms that normally maintain homeostasis of various physiological systems are likely to contribute to host tolerance to infections. Alterations in host metabolism and electrolyte balance, changes in blood pressure, impaired absorptive functions of intestinal and respiratory epithelia, local tissue hypoxia and excessive extracellular matrix remodeling, are all examples of disturbances in host physiology that can be caused by pathogens or the immune responses they elicit (17, 32). In each case, there are dedicated mechanisms that restore homeostasis and normal tissue function (regardless of what caused their dysregulation), and engagement of these mechanisms helps to reduce the fitness costs associated with infections. When these mechanisms are sufficient to prevent significant disruptions of physiological functions, infections remain asymptomatic. The pathological outcomes of infections arise when the degree of tissue damage or alteration of host physiology exceeds the capacity of tolerance mechanisms (Fig. 2). Conversely, enhancement of tolerance mechanisms should help reduce morbidity and mortality associated with infectious diseases.

\section{Tolerance capacity}

The tolerance capacity varies between different tissues and physiological processes. Four factors define the tolerance level (Fig.3) :

First, tissues and organs vary in terms of intrinsic damage susceptibility. For example, neurons and cardiomyocytes rely primarily on oxidative metabolism and therefore have low tolerance to hypoxia, compared to most other cell types that can switch to glycolysis under hypoxic conditions (17). Highly proliferative tissues, on the other hand, are more sensitive to apoptosis induced by DNA damaging agents.

Second, different tissues have different repair capacity. This capacity generally correlates with tissue renewal rates, which can range from several days (intestinal epithelium and granulocytes) to decades or even the entire life span of the organism (many neuronal cell types). Tissues with high turn-over rates, including most epithelia, and hematopoietic cell 
types, are repaired very efficiently, and therefore have higher intrinsic tolerance to damage compared to tissues with low or no renewal capacity, such as neurons and cardiomyocytes, where damage can be irreversible and often lethal.

Third, tolerance capacity depends on functional autonomy of cells that make up a given tissue. This is because functional autonomy affords compensation. Hepatocytes and RBCs have high degree of functional autonomy and therefore loss of individual cells can be compensated by the remaining cells performing the same function. The function of most neurons, on the other hand, is integrated within neuronal circuits and their target tissues. Their loss, therefore, cannot be compensated and can have devastating consequences.

Fourth, depending on a tissue, the consequences of a given degree of damage or malfunction can vary dramatically. Thus, infections with hemorrhagic viruses, such as Ebola virus or the Marburg virus, tend to be fatal because they infect and damage vascular endothelium. Extensive endothelial damage can have severe consequences because it can lead to disruption of vascular integrity, disseminated microvascular clotting and ischemia with subsequent tissue necrosis (33). Likewise, infections that cause pneumonia have high mortality rates because defects in respiratory function are poorly tolerated. In contrast, many skin infections are well tolerated and not life threatening.

The tissues that have particularly low tolerance capacity tend to be well insulated from the internal and external threats. Blood-brain barrier, skull and ribs are examples of the protective infrastructure that help prevent damage to brain, lungs and the heart - the vital organs with low tolerance to damage. One special example of the same protective strategy is the immune privileged sites, which are the tissues and organs with low tolerance to immunopathology. These tissues and organs, including brain, eye chamber, placenta and gonads, are not permissive to tissue destructive immune responses and are thus better protected from immunopathology than other tissues. They are not completely devoid of immune defensive mechanisms, however. Rather, immune privileged sites employ immune defenses that have low immunopathological potential (for example, secretory IgA and antimicrobial peptides) (34).

\section{General strategies of stress tolerance}

Cellular stress responses are inducible adaptations to adverse conditions, such as elevated temperature, ROS, osmotic pressure, endoplasmic reticulum (ER) overload, hypoxia, or exposure to noxious xenobiotics. Each of the common adverse conditions is sensed by dedicated stress response pathways that activate a transcriptional master regulator, leading to the induction of a battery of stress response genes. These genes perform various emergency functions that help the affected cells survive the adverse condition. For example, heat shock activates the transcription factor HSF-1 (heat shock factor 1) leading to the induction of genes that control refolding or degradation of misfolded proteins, thereby preventing proteotoxicity (35). Oxidative stress leads to activation of transcription factor Nrf2, which in turn induces dozens of proteins that scavenge free radicals, eliminate damaged proteins, metabolize oxidized membrane lipids and repair damaged DNA (36). ER stress activates three stress response pathway (ATF6, PERK and IRE1), which reduce new protein synthesis, eliminate misfolded proteins from the ER and restore $\mathrm{Ca}^{2+}$ and ROS homeostasis (37). In each case, a given stress stimulus activates dedicated sensors and transcription factors that induce the expression of genes required to tolerate the stressor. In the absence of the stress response pathways cells and tissues become hypersensitive to the adverse conditions. On the other hand, each stress response pathway operates at the cost of normal cell and tissue function, which is why these genes cannot be constitutively turned on and have to be inducible only when needed. 
When cellular stress response pathways are activated by a mild stressor, they become more tolerant to a more severe insult, a phenomenon known as pre-conditioning, or hormesis. The basis of pre-conditioning is the induction of protective mechanisms by a mild insult that in turn permits tolerance to a greater and potentially damaging insult. A similar phenomenon exists in the case of inflammatory tissue damage: for example, exposure to low levels of inflammatory stimuli, like bacterial lipopolysaccharide (LPS), can protect from otherwise lethal doses of LPS. This occurs through induction of negative regulators of LPS signaling and selective suppression of LPS inducible genes that have high tissue damage potential (38). Although it is not yet known to what extent this phenomenon extends to other aspects of inflammatory and immune responses, it is clear that tissue tolerance to inflammatory and other types of damage can be transiently increased upon exposure to the appropriate stressor. Moreover, it can be hypothesized that the same tolerance mechanisms can be induced by endogenous signals that report on the potential or imminent damage, before the damage actually occurred. For example, mitochondrial stress or heat shock in one tissue can induce stress adaptation in remote tissues in $C$. elegans $(39,40)$. The signals involved in stress communication presumably regulate tissue tolerance to the anticipated damage. In some contexts this type of signal is already known to exist: the protective effect against malaria afforded by sickle hemoglobin mutations results from the accumulation of free heme in plasma, a mild stressor that induces tolerance mechanisms and provides host protection against a subsequent Plasmodium induced tissue damage (28). A similar mechanism operates in severe sepsis (31). In addition, activation of the hypothalamic-pituitary-adrenal axis by anticipated threats, or by the inflammatory cytokines and tissue damage, leads to a transient reprogramming of multiple physiological processes by glucocorticoid hormones, thus promoting tolerance to the anticipated or ongoing tissue damage (41). One can expect that during different infections and other noxious insults distinct sets of tolerance promoting signals are induced to prepare the host tissues to the imminent damage associated with the particular type of infection or non-infectious insult. Identities of these signals remain to be established.

\section{Basal and inducible tolerance}

Each tissue has an intrinsic ability to tolerate some degree of stress, damage or malfunction and is thus characterized by a basal level of tolerance. The basal tolerance is afforded by constitutively active cytoprotective mechanisms and the degree of basal tolerance varies between tissues and organs. For example, cornified epithelium has higher intrinsic tolerance to damage compared to mucosal epithelium (17). The tolerance mechanisms that operate at the expense of normal tissue functions are inducible. While some cytoprotective genes are constitutively expressed at low levels, most are induced only in response to tissue stress and damage. Furthermore, some tissue protective and repair mechanisms can only operate once the damage has occurred. On the other hand, the constitutive counterpart of tissue repair, tissue renewal, is operative under basal conditions.

It should be noted that, just like a dysregulated immune response, dysregulated tolerance can lead to pathology. A notable example is fibrosis that can result from excessive tissue repair.

\section{General and specific tolerance}

Tissue protective tolerance mechanisms can be general in the sense that they are protective against most or all types of tissue stress and damage. For example, mechanisms that reduce toxic levels of ROS have cytoprotective functions for most types of stress, because ROS is elevated under most stress conditions and high levels of ROS make cells more sensitive to the damaging effects of most types of stress (42). Similarly, in some contexts, anti-apoptotic and anti-necrotic genes likely have a general tolerance enhancing effect regardless of the 
cause of cell death. Finally, tissue repair mechanisms can be expected to confer tolerance no matter the cause of tissue damage. On the other hand, specific tolerance mechanisms are only protective against some but not other forms of stress and damage. Thus, the mechanisms that maintain electrolyte balance are protective against diarrheal infections, whereas erythropoiesis is protective against hemolytic pathogens.

Activation of the general tolerance mechanisms should result in positive pre-conditioning, such that the tolerance mechanisms induced upon infection with one pathogen would increase tolerance to an unrelated pathogen. Activation of specialized tolerance mechanisms can also lead to positive pre-conditioning, as long as response to pathogen $\mathrm{A}$ is also protective against pathogen B. However, response to pathogen A can also be incompatible with the tolerance to pathogen B, resulting in a negative pre-conditioning. Some coinfections, for example, influenza virus followed by respiratory bacterial infections, result in severe morbidity and mortality. This is generally thought to be a result of compromised immunity; however, it may also be a result of compromised tolerance. Indeed it is conceivable that inducible tolerance to flu infection is incompatible with tolerance to respiratory bacterial infections. Analysis of mechanisms of pre-conditioning is important because it can provide the means to reduce morbidity and mortality by targeting the compromised tissue protective pathway(s).

\section{Sickness behavior: resistance or tolerance?}

Infections in animals and humans lead to dramatic changes in behavior, resulting in fatigue, anorexia, social withdrawal, fever and sleep alterations. Collectively these are known as sickness behaviors (43). Although sickness behavior is assumed to be adaptive, it is not clear whether and how it helps the infected host. For example, fatigue is thought to preserve energy so as to fight infection better; however, fatigue is commonly accompanied by anorexia and therefore reduced energy consumption. Fever is thought to enhance immune function, but there is little evidence of the positive effect of increased temperature on immune defenses. Fever is also thought to make the host less suitable niche for the pathogens. Different pathogens can have very different temperature preferences, however, yet fever is induced whenever an infection results in systemic inflammation. Although sickness behavior may have some undefined positive effects on the host resistance, we suggest that its benefits may have to do largely with promoting host tolerance to infection. Thus, fever may induce tissue protection by inducing HSF-1 mediated cellular heat shock response. Consistent with this view, heat shock can render cells transiently resistant to TNFmediated killing (44). Anorexia and fatigue may similarly help preserve vital processes and promote stress tolerance in multiple tissues. Indeed, anorexia has been shown to enhance tolerance to Salmonella infection in flies, while at the same time it reduces resistance to Listeria infection (45). Therefore, the presence or absence of protective effects of sickness behavior and their mechanisms are pathogen specific.

\section{Failed host defenses: Immunodeficiencies vs. deficiencies in tolerance}

Despite highly elaborate immune defense mechanisms, the host can and often does succumb to infectious diseases. Host defenses can fail because the resistance mechanisms are insufficient, overpowered or evaded by the pathogen. Alternatively, morbidity and mortality can result from the failure of tolerance mechanisms, even in the context of effective resistance. Most commonly this would be reflected in a comparable pathogen burden in hosts with different morbidity or mortality profiles. The distinction between failed resistance and failed tolerance is important because it can dictate the choice of therapeutic approaches. When the primary problem is the failed tolerance, boosting immunity and reducing pathogen burden (for example, using antibiotics or anti-malarial drugs) may be ineffective whereas 
enhancing tolerance may have salutary effects. Therapeutic or prophylactic targeting of tolerance pathways may also be the best strategy when immune defenses are either inefficient, compromised, or cause excessive immunopathology. For example, severe malaria can be effectively ameliorated, at least in experimental animals, by preventing tissue damage to liver or brain, and this protection can be uncoupled from the pathogen burden $(29,30)$. The same is true for severe sepsis, where limiting tissue damage prevents multiorgan failure, a hallmark of this disease (31). In the case of diarrheal diseases, an effective treatment is continuous re-hydration. It does not directly affect pathogen burden, but does promote host survival. Finally, flu symptoms caused by excessive inflammatory response are commonly treated by COX2 (cyclooxygenase 2) inhibitors that reduce immunopathology driven by excessive prostaglandin production, without directly affecting the pathogen burden.

Boosting tissue tolerance is also likely to be a useful strategy in the case of infectious diseases that remain a major cause of morbidity and mortality worldwide. Besides malaria, these include tuberculosis and HIV, the infectious diseases where pathogen control through vaccination or anti-microbial drugs is currently unattainable. This strategy will require identification of the tolerance mechanisms relevant to a particular infectious disease, as has been recently done for Plasmodium infections (28-30). A related strategy is the idea of an "antipathology" vaccine, as an alternative to the traditional vaccine approach that aims to reduce pathogen burden $(46,47)$. Thus, vaccination that targets and neutralizes a toxin rather than the pathogen that produces it may have a powerful therapeutic effect even without directly affecting the pathogen load. In this case, the immune system can contribute to tolerance rather than resistance (3).

Primary immunodeficiencies are commonly thought to result from mutations that affect immune functions. They may also result from defects in tolerance mechanisms, however, a possibility that is largely overlooked in both human and animal studies. To distinguish between failed resistance and failed tolerance it is important to measure pathogen burden and a relevant indicator of host fitness status. The former is not always possible in humans, however, and the latter is complicated by the fact that we almost never know what the relevant fitness characteristics are. Instead, various easily measurable indicators of health (such as body weight and temperature) are commonly used as proxies of host fitness. Systematic characterization of biomarkers of the relevant pathological processes for common infectious diseases would be useful for the development of therapies that promote host tolerance to infections.

\section{Pathogen virulence and host tolerance}

Every pathogen is characterized by a certain degree of virulence, or the ability to cause disease in a given host. Virulence can reflect either the pathogen's ability to cause direct tissue damage or the immunopathology it elicits. Highly virulent influenza viruses, for example, cause disease primarily by eliciting a "cytokine storm" - an excessive and sometimes life threatening inflammatory response (48). It is important to emphasize that virulence is a complex function that has at least two components. The pathogen-intrinsic component is defined, among other things, by the expression of toxins and other virulence factors, as well as by tissue tropism and replication rates of the pathogen. The host intrinsic component is defined by its susceptibility (or tolerance) to the damage that can be caused by the pathogen or by the immune response it elicits. Both pathogen-intrinsic and host-intrinsic characteristics can affect the host fitness (49). For example, differences in avian influenza virus tissue tropism in humans and birds are a critical determinant of virulence, which reflects the ability of influenza virus to cause immunopathology in humans and not in birds (50). Because virulence is a function of both the pathogen- and the host-intrinsic 
characteristics, it follows that the evolution of virulence can reflect changes that are either pathogen- or host-specific. Thus, a pathogen can become more virulent in a new host species and this most likely reflects the difference in the host's tolerance because pathogen-intrinsic characteristics remain unchanged. For example, the Ebola virus is highly virulent in humans because it infects and kills vascular endothelium causing lethal hemorrhages. In the natural host (which is believed to be a fruit bat) the same virus is not virulent and presumably does not cause hemorrhage (33).

In the opposite scenario, a pathogen can become less virulent in a given host because of an increase in host tolerance to damage that can be caused by the pathogen. At the extreme, a pathogen may even become a part of a normal 'commensal' microbiota, and colonize the host constitutively without causing a disease $(51,52)$. Thus, host-microbial symbiosis could be due to decreased pathogen-intrinsic virulence or due to increased host tolerance.

\section{Tolerance as a function of age}

The severity of infectious diseases can vary dramatically across different ages. Interestingly, both very young and very old are often particularly susceptible to infections. This is typically thought to reflect insufficient (immature or deteriorated) immunity in young and old, respectively. Although the immune system does deteriorate with advanced age (53), the effect of aging is more complex than a simple decline in immune resistance. For example, it is now well documented that the inflammatory and at least some immune responses in very old people can be either comparable to the young, or in some cases even enhanced (54). An alternative explanation to the higher morbidity and mortality rates in very young and very old is the compromised tolerance to infection. In babies optimal tolerance may be constrained by growth and developmental pathways. In the aged, tolerance maybe impaired because of the overall decline in tissue maintenance and repair capacities that occurs with advanced age. Frailty may be an extreme case of decline in tolerance though its causes are not well understood.

\section{Conclusions and Perspectives}

Resistance and tolerance are two alternative but complementary host defense strategies. Understanding the mechanisms that are critical for host survival is important for the choice of the therapeutic approaches. Generally, the contribution of resistance and tolerance can be distinguished by plotting pathogen burden against a health status (Fig.4) (3). It is impossible to tell the reason for morbidity and mortality when pathogen load or health status are measured alone. Because resistance by and large is the only mechanism considered in animal and human studies, when the host succumbs to infection it is usually, and often incorrectly, ascribed to the failure of the immune system.

The concept of tolerance may also be applicable to the "Typhoid Mary" phenomenon. Healthy carriers that remain asymptomatic despite being infected are likely to have a high level of tolerance to the pathogen with which they are infected. A high level of tolerance is also likely to be a characteristic of pathogen vectors. From the pathogen's perspective the vector needs to remain healthy to transmit the pathogen. Therefore, the pathogen may actually promote vector's tolerance to infection.

The concept of tolerance is not restricted to infection but can be applied to most diseases. The severity of autoimmune disease, for example, depends not only on the magnitude of self-destructive immune response, but also on the susceptibility of target tissues to the damage caused by a given level of response. It would be important to characterize the tissue protective tolerance mechanisms that help minimize negative consequences of autoimmune 
responses. Similarly, the concepts discussed here are applicable to any diseases associated with tissue damage, stress, malfunction or loss of homeostasis.

The level of tolerance to stress and tissue damage can be adjusted to the environment, at least in some animals. For example, unfavorable environments (scarce food and water sources, non-optimal temperature and humidity, etc) promote the states of suspended animation, such as spores (in protozoa), dauer (in larval stage of $C$. elegans), hibernation and estivation (a state of dormancy in animals entered in to as a response to high temperatures and arid conditions). In these states, the animals are highly tolerant to tissue damage and appear to be less susceptible to infections. Hibernating ground squirrels, for example, can sustain $-2.9^{\circ} \mathrm{C}$ core body temperature and are protected from ischemia-reperfusion injury (55). This suggests that suspended animation is associated with extreme tolerance to stress and damage and that tolerance can be regulated by environmental cues. Notably, at least in worms and flies these environmental signals are linked to the insulin growth factor-1 (IGF-1) - FoxO pathway that controls longevity (56). Defining molecular details of such pathways may pave the way to new treatment strategies for many human maladies, including infectious, inflammatory and autoimmune diseases.

\section{Acknowledgments}

The work in authors' laboratories was supported by the Howard Hughes Medical Institute (RM), grants from the NIH RO1AI055502, RO1DK071754, R37AI046688 (RM), RO1AI060164 and 1DP1OD008167-01 (DSS), NERCE (RM), Ellison Medical Foundation (DSS), Fundação para a Ciência e Tecnologia grants PTDC/SAU-TOX/ 116627/2010, PTDC/BIA-BCM/101311/2008, PTDC/SAU-FCF/100762/2008 (MS) and European Community, 6th Framework Grant, XENOME, LSH-2005-1.2.5-1 and The Bill \& Melinda Gates Foundation (MS).

\section{References and Notes}

1. Caldwell RM, Schafer JF, Compton LE, Patterson FL. Science. 1958 Sep 26.128:714. [PubMed: 17811449]

2. Schafer J. Annual Review of Phytopathology. 1971; 9:235.

3. Raberg L, Graham AL, Read AF. Philos Trans RSoc Lond B BiolSci. 2009 Jan 12.364:37.

4. Raberg L, Sim D, Read AF. Science. 2007 Nov 2.318:812. [PubMed: 17975068]

5. Read AF, Graham AL, Raberg L. PLoS Biol. 2008 Dec 23.6:e4. [PubMed: 19222305]

6. Schneider DS, Ayres JS. Nat Rev Immunol. 2008 Nov.8:889. [PubMed: 18927577]

7. Kavaliers M, Choleris E, Agmo A, Pfaff DW. Horm Behav. 2004 Sep.46:272. [PubMed: 15325228]

8. Liberles SD, et al. Proc Natl Acad Sci USA. 2009 Jun 16.106:9842. [PubMed: 19497865]

9. Riviere S, Challet L, Fluegge D, Spehr M, Rodriguez I. Nature. 2009 May 28.459:574. [PubMed: 19387439]

10. Tizzano M, et al. Proc Natl Acad Sci U S A. 2010 Feb 16.107:3210. [PubMed: 20133764]

11. Pradel E, et al. Proc Natl Acad Sci USA. 2007 Feb 13.104:2295. [PubMed: 17267603]

12. Zhang Y, Lu H, Bargmann CI. Nature. 2005 Nov 10.438:179. [PubMed: 16281027]

13. Cremer S, Sixt M. Philos Trans R Soc Lond B Biol Sci. 2009 Jan 12.364:129. [PubMed: 18926974]

14. Kiesecker JM, Skelly DK, Beard KH, Preisser E. Proc Natl Acad Sci USA. 1999 Aug 3.96:9165. [PubMed: 10430913]

15. Curtis V, de Barra M, Aunger R. Philos Trans R Soc Lond B Biol Sci. 2011 Feb 12.366:389. [PubMed: 21199843]

16. Schmid-Hempel, P. Evolutionary Parasitology. Oxford: Oxford University Press; 2011. p. 516

17. Majno, G.; Joris, I. Cells, Tissues, and Disease. Principles of General Pathology. 2nd Ed.. Oxford University Press; 2004. p. 1005

18. Graham A, Allen J, Read A. Annual Review of Ecology, Evolution and Systematics. 2005; 36:373.

19. Casadevall A, Pirofski LA. Infect Immun. 1999 Aug.67:3703. [PubMed: 10417127] 
20. Ayres JS, Freitag N, Schneider DS. Genetics. 2008 Mar.178:1807. [PubMed: 18245331]

21. Ayres JS, Schneider DS. PLoS Biol. 2008 Dec 9.6:2764. [PubMed: 19071960]

22. Cunnington AJ, de Souza JB, Walther M, Riley EM. Nat Med. 2012; 18:120. [PubMed: 22179318]

23. Shinzawa N, et al. Cell Host Microbe. 2009 Sep 17.6:244. [PubMed: 19748466]

24. Richardson CE, Kooistra T, Kim DH. Nature. 2010 Feb 25.463:1092. [PubMed: 20182512]

25. Sun J, Singh V, Kajino-Sakamoto R, Aballay A. Science. 2011 May 6.332:729. [PubMed: 21474712]

26. Ferreira A, Balla J, Jeney V, Balla G, Soares MP. J Mol Med (Berl). 2008 Oct.86:1097. [PubMed: 18641963]

27. Gozzelino R, Jeney V, Soares MP. Annu Rev Pharmacol Toxicol. 2010; 50:323. [PubMed: 20055707]

28. Ferreira A, et al. Cell. 2011 Apr 29.145:398. [PubMed: 21529713]

29. Seixas E, et al. Proc Natl Acad Sci USA. 2009 Sep 15.106:15837. [PubMed: 19706490]

30. Pamplona A, et al. Nat Med. 2007 Jun.13:703. [PubMed: 17496899]

31. Larsen R, et al. Sci Transl Med. 2010 Sep 29.51:ra71.

32. Mims, CA.; Nash, A.; Stephen, J. Mims' Pathogenesis of Infectious Disease. 5th Ed.. Academic Press; 2001. p. 474

33. Bente D, Gren J, Strong JE, Feldmann H. Dis Model Mech. 2009 Jan-Feb;2:12. [PubMed: 19132113]

34. Matzinger P, Kamala T. Nat Rev Immunol. 2011 Mar.11:221. [PubMed: 21350581]

35. Balch WE, Morimoto RI, Dillin A, Kelly JW. Science. 2008 Feb 15.319:916. [PubMed: 18276881]

36. Kensler TW, Wakabayashi N, Biswal S. Annu Rev Pharmacol Toxicol. 2007; 47:89. [PubMed: 16968214]

37. Walter P, Ron D. Science. 2011 Nov 25.334:1081. [PubMed: 22116877]

38. Foster SL, Hargreaves DC, Medzhitov R. Nature. 2007 Jun 21.447:972. [PubMed: 17538624]

39. Durieux J, Wolff S, Dillin A. Cell. 2011 Jan 7.144:79. [PubMed: 21215371]

40. Prahlad V, Cornelius T, Morimoto RI. Science. 2008 May 9.320:811. [PubMed: 18467592]

41. Sapolsky RM, Romero LM, Munck AU. Endocr Rev. 2000 Feb.21:55. [PubMed: 10696570]

42. Pamplona R, Costantini D. Am J Physiol Regul Integr Comp Physiol. 2011 Oct.301:R843. [PubMed: 21775650]

43. Hart BL. Neurosci Biobehav Rev. 1988 Summer;12:123. [PubMed: 3050629]

44. Gromkowski SH, Yagi J, Janeway CA Jr. Eur J Immunol. 1989 Sep.19:1709. [PubMed: 2792186]

45. Ayres JS, Schneider DS. PLoS Biol. 2009 Jul.7:el000150.

46. Playfair JH, Taverne J, Bate CA, de Souza JB. Immunol Today. 1990 Jan.11:25. [PubMed: 2405875]

47. Schofield L, Hewitt MC, Evans K, Siomos MA, Seeberger PH. Nature. 2002 Aug 15.418:785. [PubMed: 12181569]

48. Peiris JS, Hui KP, Yen HL. Curr Opin Immunol. 2010 Aug.22:475. [PubMed: 20594815]

49. Little TJ, Shuker DM, Colegrave N, Day T, Graham AL. PLoS Pathog. 2010; 6:e1001006. [PubMed: 20838464]

50. Tscherne DM, Garcia-Sastre A. J Clin lnvest. 2011 Jan 4.121:6.

51. Miller MR, White A, Boots M. Evolution. 2006 May.60:945. [PubMed: 16817535]

52. Roy BA, Kirchner JW. Evolution. 2000 Feb.54:51. [PubMed: 10937183]

53. Miller RA. Science. 1996 Jul 5.273:70. [PubMed: 8658199]

54. Shaw AC, Joshi S, Greenwood H, Panda A, Lord JM. Curr Opin Immunol. 2010 Aug.22:507. [PubMed: 20667703]

55. Kurtz CC, Lindell SL, Mangino MJ, Carey HV. Am]Physiol GastrointestLiver Physiol. 2006 Nov. 291:G895.

56. Kenyon CJ. Nature. 2010 Mar 25.464:504. [PubMed: 20336132] 
Tolerance to damage by pathogen
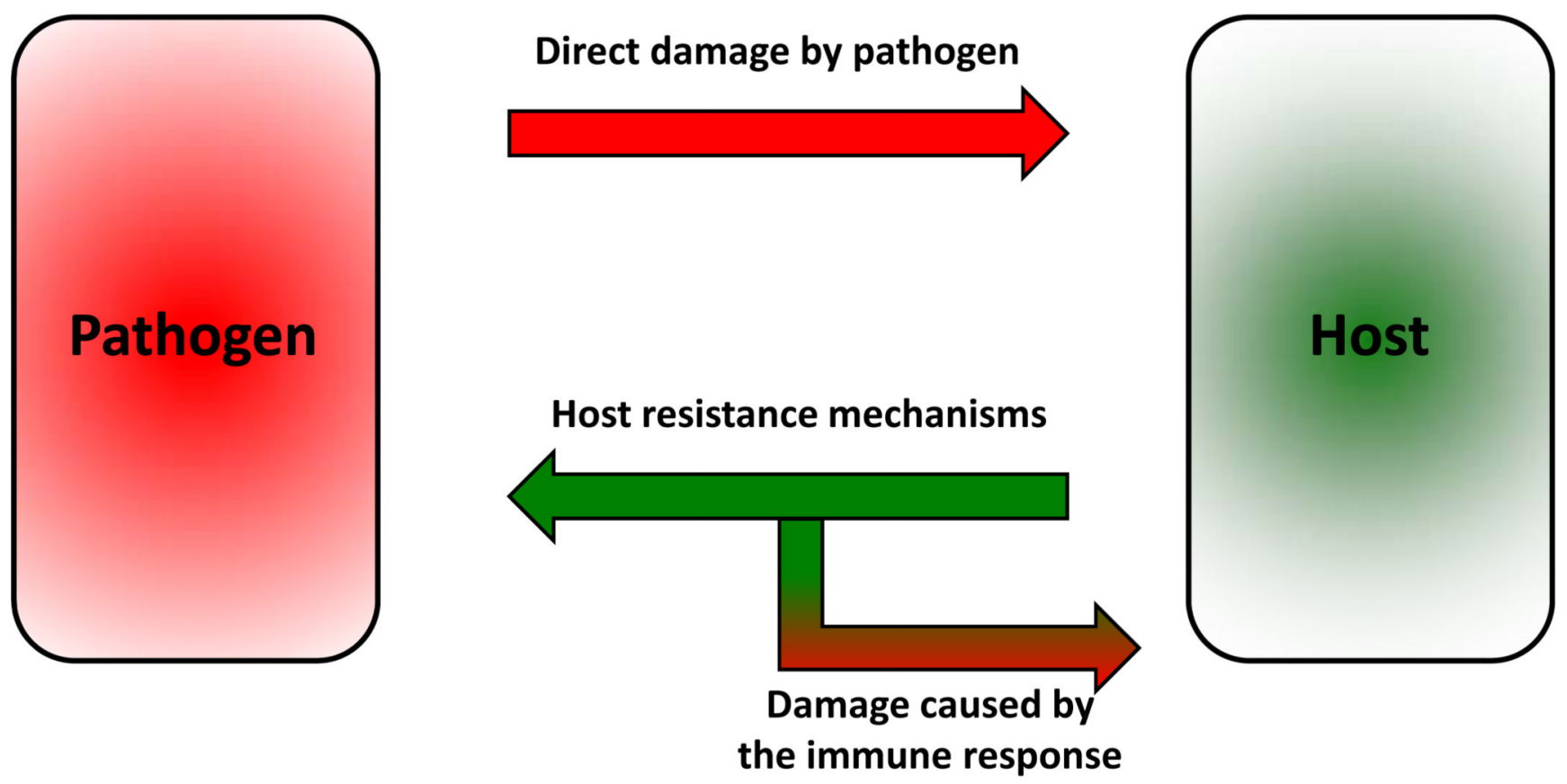

Figure 1.

Two types of fitness costs associated with infections. Pathogens can directly damage the host tissues. The immune system of the host reduces the pathogen burden through the resistance mechanism. The immune response can also damage the host tissues. The host can reduce fitness costs through tolerance mechanisms that reduce both the direct tissue damage by pathogens, and immunopathology. 


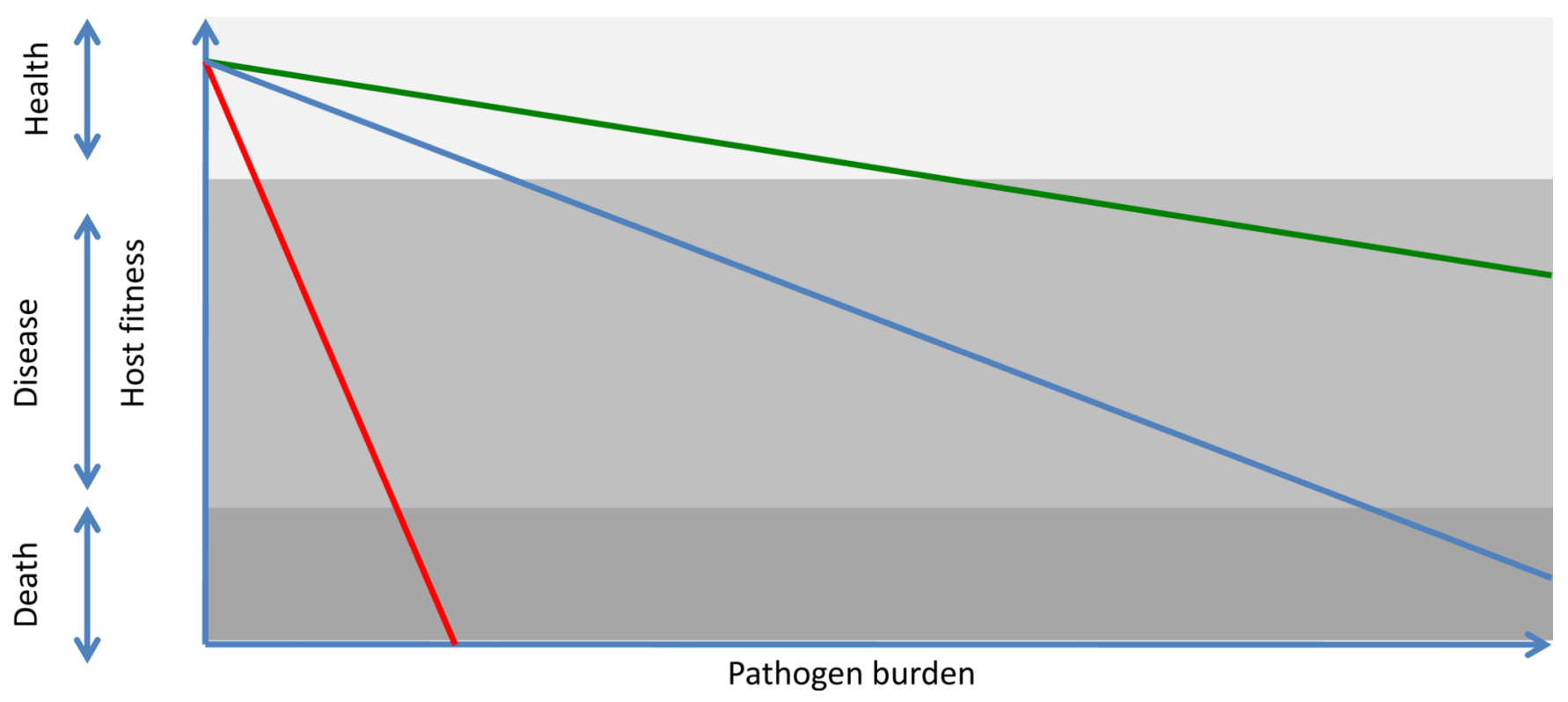

Figure 2.

Different tissues and physiological processes vary in tolerance capacity. Tissues depicted in red have the lowest tolerance to damage, the blue has an intermediate and the green has the highest tolerance capacity. 


\section{Tolerance Capacity}

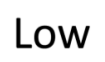

Intrinsic Damage Susceptibility

Low

Brain

Heart

Liver

Skin

Renewal \& Repair Capacity

Low

Neurons Cardiomyocytes

Hepatocytes

Intestinal Epithelium

High

Functional Autonomy

Low

Neurons Sensory Epithelia

Hepatocytes

RBCs

High

Severe

Damage Sequela

Brain Vascular \& Respiratory Systems

Liver

Skin

Moderate

Figure 3.

Tolerance capacity is a function of intrinsic damage susceptibility, repair capacity, functional autonomy, and damage sequela of different tissues and organs. Although tissues generally tend to fall at the same ends of the four spectra, the four characteristics do not necessarily correlate with each other. 


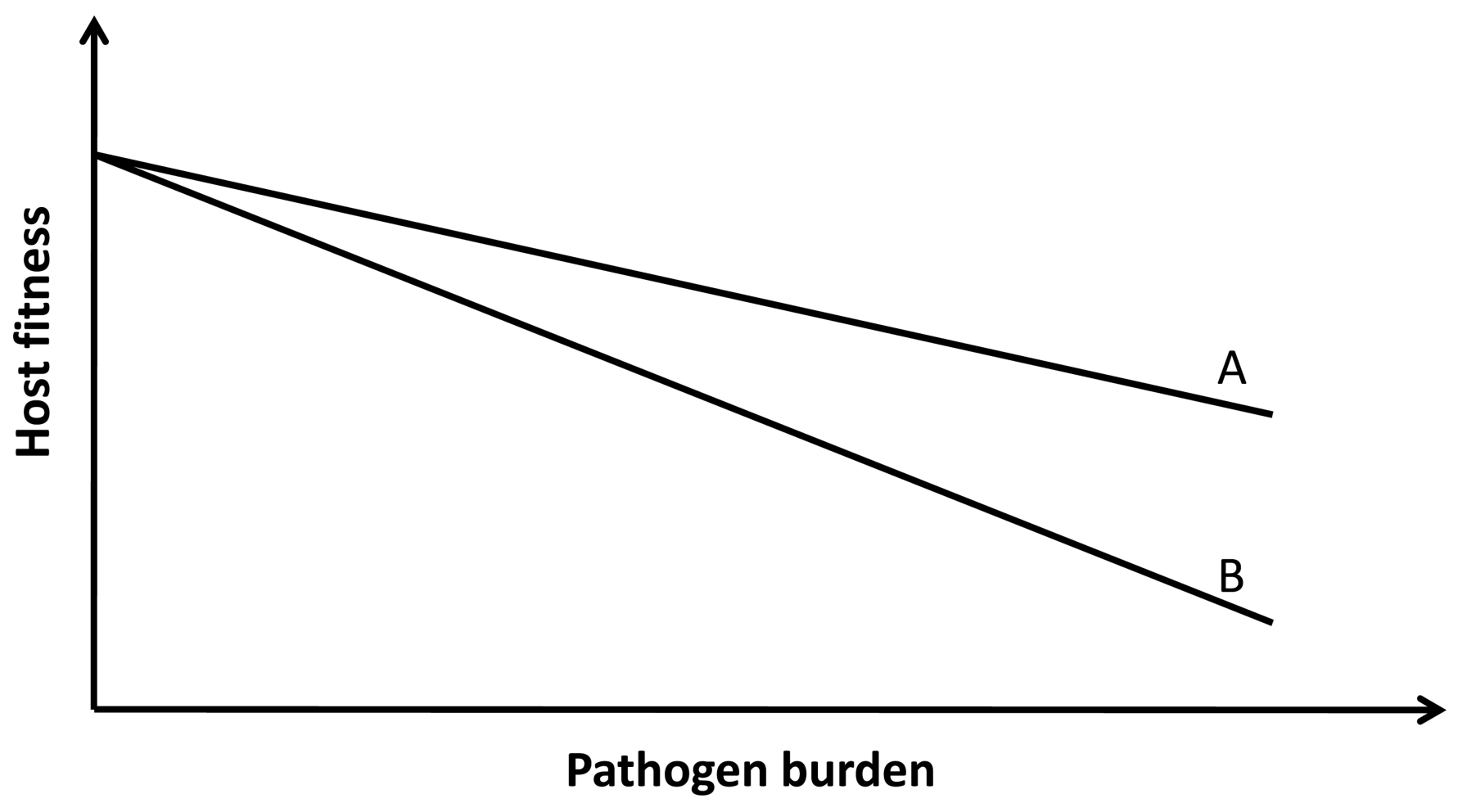

Figure 4.

When host fitness is plotted against pathogen burden, the slope of the lines reflects host tolerance to a given infection. In this example, the $\mathrm{A}$ is more tolerant to a given level of pathogen burden than B. An equivalent increase in pathogen burden will have greater negative impact on A than on B. A and B are typically different genotypes studied in the same environment. Alternatively, A and B can be two different environments where an organism with the same phenotype has different tolerance to infection. Modified from reference 3 . 